Service de Génétique et Unité de

Recherches sur les

Handicaps Génétiques de l'Enfant, INSERM U393, Hôpital des

Enfants Malades,

149 rue de Sèvres,

75743 Paris Cedex 15,

France

$S$ Gerber

D Larget-Piet

J-M Roze

A Munnich

J Kaplan

Clinique Médicale Infantile, Unité de Génétique Médicale, Hôpital Jean Bernard, 86021 Poitiers, France D Bonneau

CHRU d'Amiens,

Place Victor Pauchet, 80054 Amiens Cedex, France

M Mathieu

The Montreal Children's Hospital, McGill University, 2300 Tupper,

Montreal,

Quebec H3H 1 P3

Canada

V Der Kaloustian

Correspondence to: Dr Kaplan.

Received 21 June 1995 Revised version

accepted for publication 22 September 1995

\title{
Evidence for a fourth locus in Usher syndrome type I
}

\author{
Sylvie Gerber, Dominique Larget-Piet, Jean-Michel Rozet, Dominique Bonneau, \\ Michèle Mathieu, Vazken Der Kaloustian, Arnold Munnich, Josseline Kaplan
}

\begin{abstract}
Usher syndrome type I (US1) is an autosomal recessive condition in which three different genes have been already localised (USH1A, USH1B, and USH1C on chromosomes 14q32, 11q13, and 11 p15 respectively). The genetic heterogeneity of US1 has been confirmed in a previous study by linkage analysis of 20 French pedigrees. Here, we report the genetic exclusion of the three previously reported loci in two large multiplex families of Moroccan and Pakistani origin, suggesting the existence of at least a fourth locus in Usher syndrome type I.

( $($ Med Genet 1996;33:77-79)
\end{abstract}

Key words: Usher syndrome type I; fourth locus.

Usher syndrome type $\mathrm{I}$ is an autosomal recessive disorder characterised by profound con- genital hearing impairment with unintelligible speech, early retinitis pigmentosa, and constant vestibular dysfunction. ${ }^{1}$ Three different loci have so far been localised by linkage analysis in informative families (USH1A, USH1B, and USH1C). USH1A has been mapped to chromosome 14q32.1-14q32.3 in nine French families from the Poitou region suggesting a founder effect. ${ }^{2}$ USH1B has been mapped to chromosome 11q13.5 in 27 families from the United States, Sweden, Ireland, and South Africa $^{3}$ and USH1C has been mapped to chromosome $11 \mathrm{p} 15.1$ in eight French Acadian families. ${ }^{4}$ In 1994 , we provided evidence for genetic heterogeneity of Usher syndrome type I in 20 French pedigrees by showing that families unlinked to chromosome $14 \mathrm{q} 32$ were either linked to chromosome $11 \mathrm{q}$ or consistent with linkage to chromosome $11 \mathrm{p} .^{5}$ Here, we report the genetic exclusion of the three previously reported loci in two families of Moroccan and
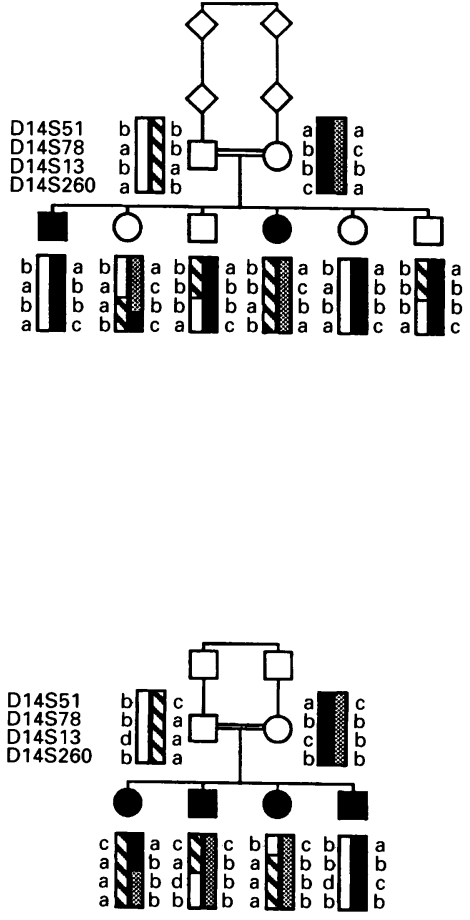

A
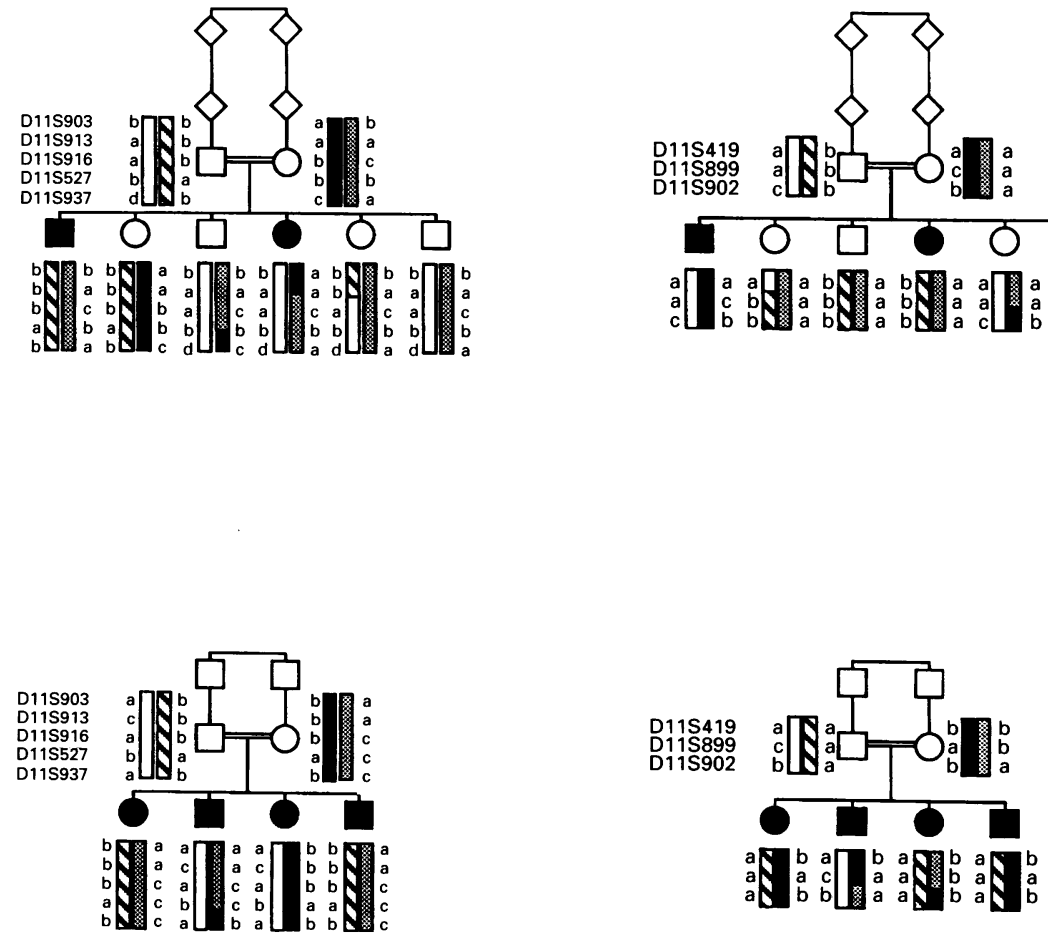

C

Figure 1 Exclusion of the Usher syndrome type I gene from chromosomes 14q32(A), 11q13(B), and 11p15(C) in both families. 
Pairwise lod score values for polymorphic markers of chromosome $14 q, 11 q$, and $11 p$

\begin{tabular}{|c|c|c|c|c|c|c|c|c|c|}
\hline \multirow[t]{2}{*}{ Locus (probe) } & \multirow[t]{2}{*}{ Families } & \multicolumn{6}{|c|}{ Recombination fractions } & \multirow[b]{2}{*}{$Z$} & \multirow[b]{2}{*}{$\theta$} \\
\hline & & 0 & 0.01 & $0 \cdot 05$ & $0 \cdot 10$ & $0 \cdot 20$ & $0 \cdot 30$ & & \\
\hline $\begin{array}{l}\text { Chromosome } 14 q \\
\text { D14S51 } \\
\text { (mfd165) }\end{array}$ & $\begin{array}{l}\text { Fam 1 } \\
\text { Fam } 2 \\
\text { Total }\end{array}$ & $\begin{array}{l}-0 \cdot 63 \\
-\infty \\
-\infty\end{array}$ & $\begin{array}{l}-0.55 \\
-5 \cdot 73 \\
-6 \cdot 28\end{array}$ & $\begin{array}{l}-0.35 \\
-2 \cdot 98 \\
-3 \cdot 33\end{array}$ & $\begin{array}{l}-0.22 \\
-1 \cdot 84 \\
-2 \cdot 06\end{array}$ & $\begin{array}{l}-0.09 \\
-0.81 \\
-0.90\end{array}$ & $\begin{array}{l}-0.04 \\
-0.31 \\
-0.35\end{array}$ & $\begin{array}{l}0 \\
0 \\
0\end{array}$ & $\begin{array}{l}0 \cdot 50 \\
0 \cdot 50 \\
0.50\end{array}$ \\
\hline $\begin{array}{l}\text { D14S78 } \\
\text { (AFM234we5) }\end{array}$ & $\begin{array}{l}\text { Fam } 1 \\
\text { Fam 2 } \\
\text { Total }\end{array}$ & $\begin{array}{l}-\infty \\
-0 \cdot 37 \\
-\infty\end{array}$ & $\begin{array}{l}-3 \cdot 47 \\
-0.67 \\
-3 \cdot 64\end{array}$ & $\begin{array}{r}-1 \cdot 88 \\
0 \cdot 15 \\
-1 \cdot 73\end{array}$ & $\begin{array}{r}-1 \cdot 16 \\
0 \cdot 26 \\
-0 \cdot 89\end{array}$ & $\begin{array}{r}-0 \cdot 50 \\
0 \cdot 25 \\
-0 \cdot 25\end{array}$ & $\begin{array}{r}-0.19 \\
0.13 \\
-0.06\end{array}$ & $\begin{array}{l}0 \\
0 \cdot 26 \\
0\end{array}$ & $\begin{array}{l}0 \cdot 50 \\
0 \cdot 10 \\
0 \cdot 50\end{array}$ \\
\hline $\begin{array}{l}\text { D14S13 } \\
\text { (MLJ14) }\end{array}$ & $\begin{array}{l}\text { Fam 1 } \\
\text { Fam } 2 \\
\text { Total }\end{array}$ & $\begin{array}{l}-\infty \\
-\infty \\
-\infty\end{array}$ & $\begin{array}{l}-1.54 \\
-4.87 \\
-6.41\end{array}$ & $\begin{array}{l}-0 \cdot 81 \\
-2 \cdot 44 \\
-3 \cdot 25\end{array}$ & $\begin{array}{l}-0.32 \\
-0.91 \\
-1.93\end{array}$ & $\begin{array}{l}-0.20 \\
-0.58 \\
-0.78\end{array}$ & $\begin{array}{l}-0.07 \\
-0.21 \\
-0.28\end{array}$ & $\begin{array}{l}0 \\
0 \\
0\end{array}$ & $\begin{array}{l}0.50 \\
0.50 \\
0.50\end{array}$ \\
\hline $\begin{array}{l}\text { D14S260 } \\
\text { (AFM238we7) }\end{array}$ & $\begin{array}{l}\text { Fam } 1 \\
\text { Fam 2 } \\
\text { Total }\end{array}$ & $\begin{array}{l}-\infty \\
-\infty \\
-\infty\end{array}$ & $\begin{array}{l}-2 \cdot 54 \\
-2 \cdot 70 \\
-5 \cdot 24\end{array}$ & $\begin{array}{l}-1 \cdot 27 \\
-1 \cdot 36 \\
-2 \cdot 63\end{array}$ & $\begin{array}{l}-0.80 \\
-0.82 \\
-1.62\end{array}$ & $\begin{array}{l}-0.38 \\
-0.36 \\
-0.74\end{array}$ & $\begin{array}{l}-0 \cdot 16 \\
-0 \cdot 14 \\
-0 \cdot 30\end{array}$ & $\begin{array}{l}0 \\
0 \\
0\end{array}$ & $\begin{array}{l}0.50 \\
0.50 \\
0.50\end{array}$ \\
\hline $\begin{array}{l}\text { Chromosome } 11 q \\
\text { D11S903 } \\
\text { (AFM077xel) }\end{array}$ & $\begin{array}{l}\text { Fam } 1 \\
\text { Fam } 2 \\
\text { Total }\end{array}$ & $\begin{array}{l}-\infty \\
-\infty \\
-\infty\end{array}$ & $\begin{array}{l}-1.91 \\
-1.65 \\
-3.56\end{array}$ & $\begin{array}{l}-0.89 \\
-0.87 \\
-1 \cdot 76\end{array}$ & $\begin{array}{l}-0.55 \\
-0.45 \\
-1.00\end{array}$ & $\begin{array}{l}-0 \cdot 23 \\
-0 \cdot 15 \\
-0.38\end{array}$ & $\begin{array}{l}-0.09 \\
-0.05 \\
-0 \cdot 14\end{array}$ & $\begin{array}{l}0 \\
0 \\
0\end{array}$ & $\begin{array}{l}0 \cdot 50 \\
0 \cdot 50 \\
0 \cdot 50\end{array}$ \\
\hline $\begin{array}{l}\text { D11S913 } \\
\text { (AFM164zf12) }\end{array}$ & $\begin{array}{l}\text { Fam } 1 \\
\text { Fam 2 } \\
\text { Total }\end{array}$ & $\begin{array}{l}-\infty \\
-\infty \\
-\infty\end{array}$ & $\begin{array}{l}-1.43 \\
-4.93 \\
-6.36\end{array}$ & $\begin{array}{l}-0 \cdot 75 \\
-2 \cdot 51 \\
-3 \cdot 26\end{array}$ & $\begin{array}{l}-0.47 \\
-1.50 \\
-1.97\end{array}$ & $\begin{array}{l}-0.21 \\
-0.63 \\
-0.84\end{array}$ & $\begin{array}{l}-0.08 \\
-0.25 \\
-0.33\end{array}$ & $\begin{array}{l}0 \\
0 \\
0\end{array}$ & $\begin{array}{l}0.50 \\
0.50 \\
0.50\end{array}$ \\
\hline $\begin{array}{l}\text { D11S916 } \\
\text { (AFM185yal) }\end{array}$ & $\begin{array}{l}\text { Fam 1 } \\
\text { Fam 2 } \\
\text { Total }\end{array}$ & $\begin{array}{l}-\infty \\
-\infty \\
-\infty\end{array}$ & $\begin{array}{l}-1.50 \\
-4.92 \\
-6.42\end{array}$ & $\begin{array}{l}-0 \cdot 71 \\
-2 \cdot 51 \\
-3 \cdot 22\end{array}$ & $\begin{array}{l}-0.42 \\
-1.50 \\
-1.92\end{array}$ & $\begin{array}{l}-0 \cdot 21 \\
-0 \cdot 63 \\
-0 \cdot 84\end{array}$ & $\begin{array}{l}-0 \cdot 10 \\
-0 \cdot 25 \\
-0.35\end{array}$ & $\begin{array}{l}0 \\
0 \\
0\end{array}$ & $\begin{array}{l}0 \cdot 50 \\
0 \cdot 50 \\
0 \cdot 50\end{array}$ \\
\hline $\begin{array}{l}\text { D11S527 } \\
\text { (zd5) }\end{array}$ & $\begin{array}{l}\text { Fam } 1 \\
\text { Fam } 2 \\
\text { Total }\end{array}$ & $\begin{array}{l}-\infty \\
-\infty \\
-\infty\end{array}$ & $\begin{array}{l}-1.60 \\
-4.93 \\
-6.53\end{array}$ & $\begin{array}{l}-0.85 \\
-2.51 \\
-3.36\end{array}$ & $\begin{array}{l}-0.53 \\
-1.50 \\
-2.03\end{array}$ & $\begin{array}{l}-0.22 \\
-0.63 \\
-0.85\end{array}$ & $\begin{array}{l}-0.08 \\
-0.25 \\
-0.33\end{array}$ & $\begin{array}{l}0 \\
0 \\
0\end{array}$ & $\begin{array}{l}0 \cdot 50 \\
0 \cdot 50 \\
0.50\end{array}$ \\
\hline $\begin{array}{l}\text { D11S937 } \\
\text { (AFM256zb5) }\end{array}$ & $\begin{array}{l}\text { Fam } 1 \\
\text { Fam 2 } \\
\text { Total }\end{array}$ & $\begin{array}{l}-\infty \\
-\infty \\
-\infty\end{array}$ & $\begin{array}{l}-1.48 \\
-5.66 \\
-7.14\end{array}$ & $\begin{array}{l}-0.67 \\
-2.93 \\
-3.60\end{array}$ & $\begin{array}{l}-0.36 \\
-1.80 \\
-2.16\end{array}$ & $\begin{array}{l}-0.13 \\
-0.79 \\
-0.92\end{array}$ & $\begin{array}{l}-0.05 \\
-0.31 \\
-0.36\end{array}$ & $\begin{array}{l}0 \\
0 \\
0\end{array}$ & $\begin{array}{l}0 \cdot 50 \\
0 \cdot 50 \\
0 \cdot 50\end{array}$ \\
\hline $\begin{array}{l}\text { Chromosome 11p } \\
\text { D11S419 } \\
\text { (Mfd58) }\end{array}$ & $\begin{array}{l}\text { Fam 1 } \\
\text { Fam 2 } \\
\text { Total }\end{array}$ & $\begin{array}{l}-\infty \\
-1 \cdot 22 \\
-\infty\end{array}$ & $\begin{array}{l}-1 \cdot 65 \\
-0.96 \\
-2.61\end{array}$ & $\begin{array}{l}-0 \cdot 89 \\
-0.54 \\
-1 \cdot 43\end{array}$ & $\begin{array}{l}-0.55 \\
-0.32 \\
-0.87\end{array}$ & $\begin{array}{l}-0 \cdot 23 \\
-0 \cdot 13 \\
-0.36\end{array}$ & $\begin{array}{l}-0.09 \\
-0.05 \\
-0.14\end{array}$ & $\begin{array}{l}0 \\
0 \\
0\end{array}$ & $\begin{array}{l}0 \cdot 50 \\
0 \cdot 50 \\
0 \cdot 50\end{array}$ \\
\hline $\begin{array}{l}\text { D11S899 } \\
\text { (AFM022th2) }\end{array}$ & $\begin{array}{l}\text { Fam 1 } \\
\text { Fam 2 } \\
\text { Total }\end{array}$ & $\begin{array}{l}-\infty \\
-\infty \\
-\infty\end{array}$ & $\begin{array}{l}-3 \cdot 40 \\
-2 \cdot 34 \\
-5 \cdot 74\end{array}$ & $\begin{array}{l}-1 \cdot 76 \\
-1 \cdot 06 \\
-2 \cdot 82\end{array}$ & $\begin{array}{l}-1.07 \\
-0.59 \\
-1.66\end{array}$ & $\begin{array}{l}-0.46 \\
-0.22 \\
-0.67\end{array}$ & $\begin{array}{l}-0.18 \\
-0.07 \\
-0.25\end{array}$ & $\begin{array}{l}0 \\
0 \\
0\end{array}$ & $\begin{array}{l}0 \cdot 50 \\
0 \cdot 50 \\
0 \cdot 50\end{array}$ \\
\hline $\begin{array}{l}\text { D11S902 } \\
\text { (AFM072yd3) }\end{array}$ & $\begin{array}{l}\text { Fam 1 } \\
\text { Fam 2 } \\
\text { Total }\end{array}$ & $\begin{array}{l}-\infty \\
-0 \cdot 47 \\
-\infty\end{array}$ & $\begin{array}{l}-2 \cdot 54 \\
-0 \cdot 24 \\
-2 \cdot 78\end{array}$ & $\begin{array}{r}-1 \cdot 26 \\
0 \cdot 04 \\
-1 \cdot 21\end{array}$ & $\begin{array}{r}-0 \cdot 79 \\
0 \cdot 10 \\
-0 \cdot 69\end{array}$ & $\begin{array}{r}-0.39 \\
0.02 \\
-0.37\end{array}$ & $\begin{array}{l}-0.17 \\
-0.03 \\
-0.20\end{array}$ & $\begin{array}{l}0 \\
0 \cdot 10 \\
0\end{array}$ & $\begin{array}{l}0 \cdot 50 \\
0 \cdot 10 \\
0 \cdot 50 \\
\end{array}$ \\
\hline
\end{tabular}

Pakistani ancestry and suggest the existence of a fourth locus in Usher syndrome type I.

Six affected subjects and eight healthy relatives belonging to two consanguineous families of Moroccan and Pakistani origin, respectively, were ascertained. The affected subjects fulfilled the inclusion criteria of Usher syndrome type $I$, that is (1) deafness and absence of spontaneous language discovered in the first 18 months of life; (2) evidence of retinitis pigmentosa, including extinction of electroretinogram (ERG) before 10 years of age when systematically looked for and consistently present within the first two decades; and (3) bilateral vestibular areflexia (caloric test and rotational tests ${ }^{2}$ ). Genotyping at the three Usher syndrome type I loci was carried out as previously described. ${ }^{2-4}$

The table shows that consistently negative pairwise lod score values were obtained for polymorphic markers of chromosomes $14 \mathrm{q}$, $11 \mathrm{q}$, and $11 \mathrm{p}$. Multipoint linkage analyses showed a large exclusion area around USH1A, USH1B, USH1C $(40,45$, and $30 \mathrm{cM}$ respectively) (fig 1) and haplotype analyses confirmed the unambiguous exclusion of the three Usher syndrome type I loci (fig 2).
Usher syndrome has long been regarded as a clinically and genetically heterogeneous condition. Indeed, at least two genes account for the late onset form of the disease (Usher syndrome type II, chromosome $1 \mathrm{q} 42,{ }^{6}$ and an as yet unlocalised second locus, ${ }^{7}$ while at least four genes now account for Usher syndrome type I. This figure is not unusual in autosomal recessive retinal dystrophies. For example, despite its clinical homogeneity, the Bardet-Biedl syndrome is now known to be accounted for by at least five genes. ${ }^{8-12}$

By providing evidence of at least a fourth locus, the present study gives additional support to the genetic heterogeneity of Usher syndrome type I. This genetic heterogeneity hampers prenatal diagnosis of this severe condition in small pedigrees and makes the identification of the gene particularly difficult. Yet, the identification of the myosin VIIA gene as the USH1B gene will hopefully speed up the identification of the other Usher syndrome genes. ${ }^{13}$

We thank Gisèle Gal for her help in preparing this manuscript. This study was supported by the Association Française contre les Myopathies (AFM), Association Française Retinitis Pigmentosa (AFRP), and the Groupement d'Etudes et de Recherches sur les Génomes (GREG). 


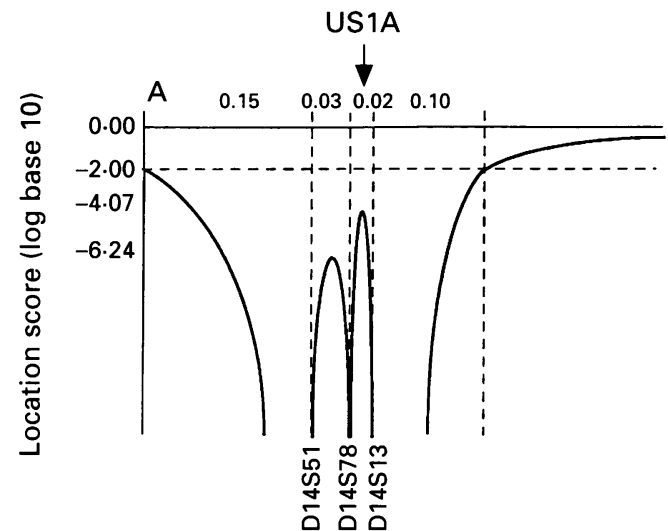

Genetic location (cM) on chromosome 14q

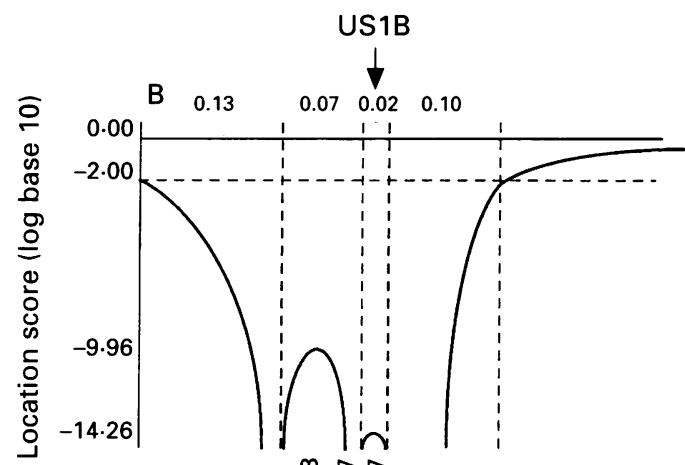

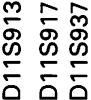

Genetic location (cM) on chromosome 11q

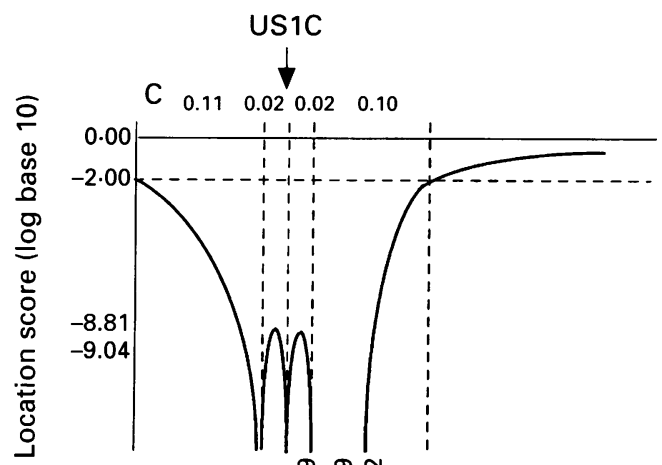

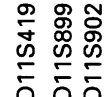

Genetic location (cM) on chromosome 11p
1 Usher $\mathrm{CH}$. On the inheritance of retinitis pigmentosa with notes of cases. $R$ Lond Ophthalmol Hosp Rep 1913/14;19: $130-236$

2 Kaplan J, Gerber S, Bonneau D, et al. A gene for Usher syndrome type I maps to chromosome $14 \mathrm{q}$. Genomics 1992;14:979-87.

3 Kimberling WJ, Moller CG, Davenport S, et al. Linkage of Usher syndrome type I gene (USH1B) to the long arm of chromosome 11. Genomics 1992;14:988-94.

4 Smith RJH, Lee EC, Kimberling WJ, et al. Localization of two genes for Usher syndrome type 1 to chromosome 11 . Genomics 1992;14:995-1002.

5 Larget-Piet D, Gerber S, Bonneau D, et al. Genetic heterogeneity of Usher syndrome type I in French families. Genomics 1994;21:138-43.

6 Kimberling WJ, Weston MD, Moller C, et al. Localization of Usher syndrome type II to chromosome 1q. Genomics of Usher syndrome type II to chromosome 1q. Genomics

7 Dahl PS, Kimberling WJ, Gorin MB, et al. Genetic heterogeneity of Usher syndrome type II. F Med Genet 1993;30: $843-8$.

8 Kwitek-Black AE, Carmi R, Duyk GM, et al. Linkage of Bardet-Biedl syndrome to chromosome $16 \mathrm{q}$ and evidence for non-allelic genetic heterogeneity. Nature Genet 1993; 5:392-6.

9 Leppert M, Baird L, Anderson KL, Otterud B, Lupski JR, Lewis RA. Bardet-Biedl syndrome is linked to DNA markers on chromosome $11 \mathrm{q}$ and is genetically heterogeneous. Nature Genet 1994;7:108-11.

10 Sheffield VC, Carmi R, Kwitek-Black AE, et al. Identification of a Bardet Biedl syndrome locus on chromosome 3 and evaluation of an efficient approach to homozygosity 3 and evaluation of an efficient approach

11 Carmi R, Rokhlina T, Kwitek-Black A, et al. Use of a DNA pooling strategy to identify a human obesity syndrome pooling strategy to identify a human obesity syndrome 2 Sunden SLF, Heon E, Kwitek-Black AE, et al. Genetic Sunden SLF, Heon E, Kwitek-Black AE, et al. Genetic
heterogeneity in 23 Canadian Bardet-Biedl families and heterogeneity in 23 Canadian Bardet-Biedl families and
evidence for a fifth BBS locus. Invest Ophthalmol Vis Sci 1995;36:A3582, abstract book.

13 Weil D, Blanchard S, Kaplan J, et al. Defective myosin VIIA gene responsible for Usher syndrome type 1B. Nature 1995;374:60-1.

Figure 2 Segregation of marker alleles of chromosomes $14 q(A), 11 q(B)$, and $11 p(C)$ in families 1 and 2. 